\title{
Non-Invasive Microwave Sensors for Biomedical Applications: New Design Perspectives
}

\author{
Sandra COSTANZO \\ DIMES, University of Calabria, Via P. Bucci cubo 42C, 87036 Rende (CS), Italy \\ costanzo@dimes.unical.it
}

Submitted May 1, 2017 / Accepted May 1, 2017

\begin{abstract}
The basic operation principles of non-invasive microwave sensors are summarized in this work, with specific emphasis on health-care systems applications. Design criteria to achieve reliable results in terms of biological parameters detection are specifically highlighted. In particular, the importance to adopt accurate frequency models for the complex permittivity (in terms of both dielectric constant as well as loss tangent) in the synthesis procedure of the microwave sensor is clearly motivated. Finally, an application example of the outlined new perspectives in the framework of glucose monitoring to face diabete disease is deeply discussed.
\end{abstract}

\section{Keywords}

Microwaves, sensors, biomedical applications, dielectric characterization

\section{Introduction}

The increase in chronic pathologies related to actual lifestyle demands for urgent changes in the evolution of health-care systems. A primary challenge to satisfy this need is the achievement of high-care at reduced costs, by focusing on the promotion of prevention and effective disease management, rather than on specialized care systems for the treatment of late-stage pathologies. Healthcare providers and users are increasingly interested in the adoption of new communication technologies (e.g. smartphones), thus moving towards a new paradigm of 'e-health' monitoring (Fig. 1), which however is at an early stage and is still in development. In order to provide accurate and early diagnoses, e-health devices should guarantee a continuous monitoring without interference in daily life, and they should also avoid the need of external people for the biomedical control. To this end, microwave biosensors can give a valid alternative to standard chemical devices, typically having short lifetime, and based on the use of fluid probe which limits the application to discontinuous monitoring.

The basic principle of microwave biosensors relies on the specific property of electromagnetic fields to interact

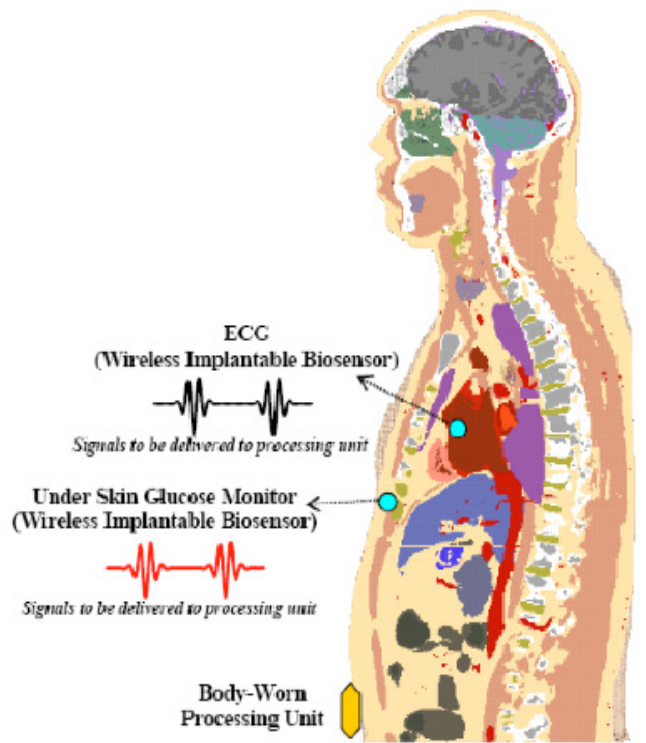

Fig. 1. A description of e-health monitoring (courtesy of http://antennas.eecs.qmul.ac.uk/research/body-centricwireless-commnication-and-networks/)

with matter, in a different way depending on its molecular structure, thus leading to investigate the tissues compositions by examining the variation of their dielectric properties in response to the applied excitation field.

Specific advantages can be identified in the adoption of microwaves and millimeter waves. First of all, the ability of electromagnetic fields to penetrate into biological media can be exploited to implement noninvasive measurements. Furthermore, microwaves represent nonionizing fields, thus avoiding dangers related to the adoption of other types of radiation, such as X-rays. When exposed to microwave fields, biological molecules become polarized similarly to water, and the excitation wave travels more slowly with respect to a free-space environment. Microwave biosensors are so designed to convert these changes in the wave propagation speed through the biological medium into a quantifiable signal giving the variation of a specific bio-parameter.

In its practical configuration, a microwave biosensor is composed by two specific parts, namely the microwave sensor element and the probe circuit. Various architectures can be adopted to implement this latter component, which 
is essentially demanded at replacing in a compact miniaturized form the functionality of a vector network analyzer (VNA). Practically, it should be able to characterize the propagation features of the microwave sensor in the presence of the biological medium, in order to extract its dielectric properties. Since the early 1970s, various architectures have been introduced in literature, such as those based on heterodyne architecture [1] or the six-port technique [2].

All existing approaches are well suitable to be soon applied in a large class of biomedical applications [3-6], by also exploiting emerging integration techniques to realize devices in a very compact form. However, further advancements are still required for a more selective correlation of the different stages of a specific pathology to the changes in the dielectric features of the involved biological medium. These challenging advancements directly involve a more accurate design of the microwave sensor element, through the development of more reliable dielectric models for biomaterials, to be successively translated into more reliable correlations to the different stages of the monitored disease.

\section{Basic Principle and Design Criteria}

The ability of microwave sensors to monitoring biological parameters relies on the fact that all biological molecules have a different dielectric behavior as compared to air or water, thus changes in the wave propagation speed (or, equivalently, in the dielectric permittivity) can be in principle correlated to the human tissues compositions. However, to achieve reliable results in terms of biological parameter changes, accurate models are strongly required to define the variation of dielectric permittivity versus frequency for different biological materials. Actually, only approximated models exist, essentially derived from empirical evaluations.

At microwave and millimeter-wave frequencies, the interaction between electromagnetic field and matter caused two specific phenomena, namely:

- a reorientation motion of molecular dipoles, which is a polarization effect modeled by the real part of permittivity;

- a translational motion of free electric charges, which is modeled by an equivalent conductivity, giving rise to an imaginary part in the permittivity.

For polar molecules such as water, the frequency behavior of permittivity is described in literature by the Debye equation as [7]:

$$
\varepsilon_{\mathrm{r}}=\varepsilon_{\infty}+\frac{\varepsilon_{\mathrm{s}}-\varepsilon_{\infty}}{1+\mathrm{j} \omega \tau}+\frac{\sigma}{\mathrm{j} \omega \varepsilon_{\mathrm{o}}}
$$

where $\varepsilon_{\mathrm{s}}$ is the low-frequency permittivity; $\varepsilon_{\infty}$ is the highfrequency permittivity; $\tau$ gives the relaxation time; $\sigma$ represents the ionic conductivity; $\varepsilon_{0}$ is the free-space permittivity.

Even if biological materials contain large quantities of water (typically $70-80 \%$ ), their dielectric properties at microwave and millimeter-wave frequencies exhibit a behavior which differs from the simple model given by (1). So, to take into account for the different relaxation processes, the Cole-Cole model can be adopted for an efficient dielectric representation of biological tissues over frequency, as given by the following expression [8]:

$$
\varepsilon_{\mathrm{r}}=\varepsilon_{\infty}+\frac{\varepsilon_{\mathrm{s}}-\varepsilon_{\infty}}{1+(\mathrm{j} \omega \tau)^{1-\alpha}}+\frac{\sigma}{j \omega \varepsilon_{\mathrm{o}}}
$$

where the new parameter $\alpha$ models the broadening of the dispersion lines.

For better approximations, a description in terms of multiple Cole-Cole dispersion can be adopted, as given by [8]:

$$
\varepsilon_{\mathrm{r}}=\varepsilon_{\infty}+\sum_{n} \frac{\varepsilon_{\mathrm{s} n}-\varepsilon_{\infty}}{1+\left(\mathrm{j} \omega \tau_{n}\right)^{1-\alpha}}+\frac{\sigma}{\mathrm{j} \omega \varepsilon_{\mathrm{o}}} .
$$

Most of existing works on microwave biosensors do not assume the complete form of the Cole-Cole model, but the ionic conductivity $\sigma$, giving rise to the loss tangent, is typically neglected. This causes an important approximation error in the retrieval of biological parameter.

The adoption of accurate dielectric models for the tissue structure to be monitored has a fundamental importance to retrieve reliable information and diagnose pathologies at an early stage. As a matter of fact, the microwave sensor should be designed by properly taking into account the lossy behavior of the specific biological material to be monitored, which acts as radiation medium. This will lead to establish a reliable relationship between the response of the microwave sensor and the biological parameter to be detected for health-care purposes. However, some specific points still remain open to further advancements. First of all, most of existing microwave biosensors in literature are designed on the basis of full-wave simulations performed through cad software, with the optimization achieved numerically and typically assuming only the frequency variation of the real part of permittivity for different tissues structures. To improve accuracy, the variation of the imaginary part of permittivity should be also included in the simulation model, and the design process of the microwave sensor should be enhanced by adopting analytical models which assume the biological material as lossy radiation medium. On the other side, further developments should be also performed to investigate dielectric models for inhomogeneous biomaterials, thus improving the accuracy of future implementation of microwave biosensors. 


\section{Application Example: Glucose Monitoring}

Diabete is a chronic disease resulting in the inability of affected patients to control the glucose level in the blood stream. The increasing aging of population and the rise of obesity negatively act to a continuous increment in the number of diabete patients, and a proper monitoring of blood glucose levels is strongly required for managing the disease and avoiding complications.

To achieve a continuous monitoring of glucose levels, implantable biosensors have been introduced [6-9] which rely on the interstitial fluid within the dermis to perform the measurement. However, many degradation factors, including also tissue fibrosis and inflammation, lead to a very limited implantation time ( $\max 10$ days), thus turning the attention of research to the investigation of alternative non-invasive procedures. To this end, various configurations of microwave sensors have been proposed in literature, all based on the principle that blood permittivity is affected by its glucose concentration, so glucose levels can be retrieved from the detection of frequency shift in the reflection response of the resonant microwave sensor. Many papers can be found which prove the feasibility of non-invasive blood glucose monitoring through the adoption of a resonant antenna [5], [10-12], but further work is still required to achieve true reliable implementations.

A primary approximation of existing approaches relies on the fact that the adopted design procedure only assumes the frequency variation of the real part of permittivity, while neglecting the effect of glucose concentration on the loss tangent of blood. This important aspect has been recently faced by the author in a preliminary work [13] and it is detailed here as illustration example.

To demonstrate the concept, a very simple configuration is assumed for the resonant microwave sensor, namely a standard inset-fed patch antenna working in the Industrial, Scientific, Medical (ISM) band around a frequency $f_{\mathrm{o}}=2.4 \mathrm{GHz}$. A high permittivity dielectric $\left(\varepsilon_{\mathrm{r}}=10\right)$ is chosen as antenna substrate, in order to reduce the effect of environmental properties as much as possible. The optimization of antenna dimensions is performed on Ansys software to achieve the resonant condition at the design frequency $f_{\mathrm{o}}$, but considering the proper frequency variation of the complex permittivity (dielectric constant as well as loss tangent) relative to the biological material, which acts as radiation medium.

As the objective is the demonstration of enhanced design principle, a water-glucose solution is assumed as radiation environment for this preliminary validation work. First, an experimental characterization of complex permittivity is performed on water solutions with different glucose concentrations (GC). Dielectric measurements are executed in the Microwave Laboratory at University of Calabria, by adopting the Anritsu VectorStar VNA and the open-ended coaxial probe Speag DAK, with an uncertainty

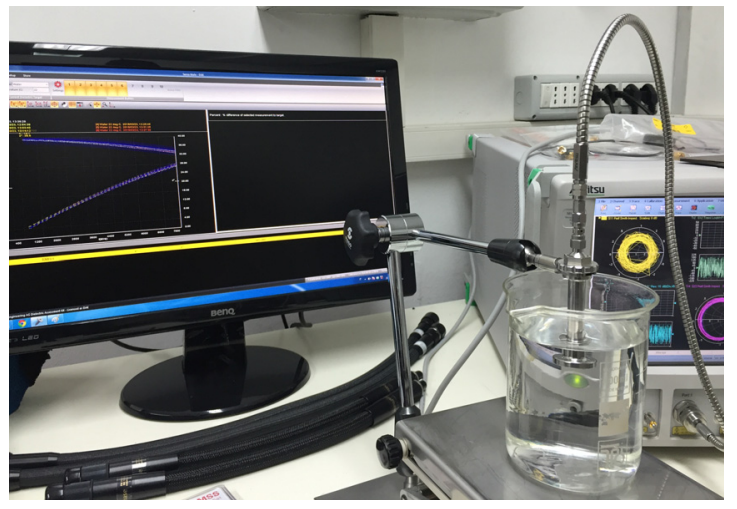

Fig. 2. Test setup for dielectric measurements (Microwave Laboratory at University of Calabria).

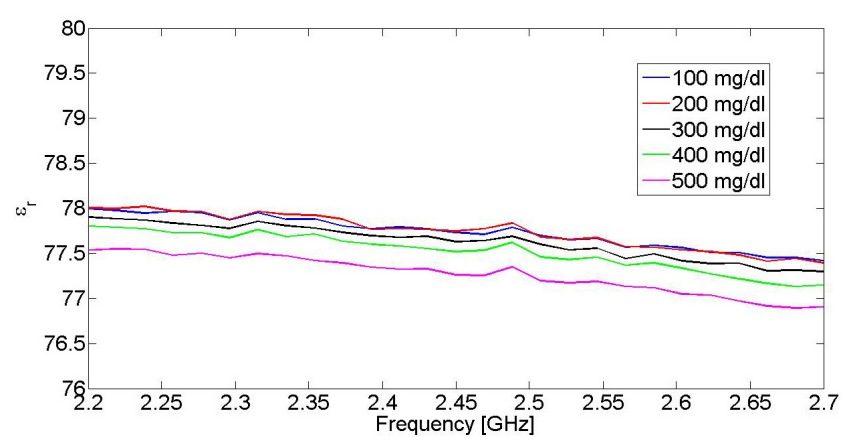

Fig. 3. Measured dielectric constant vs. frequency for water solutions with different glucose concentrations.

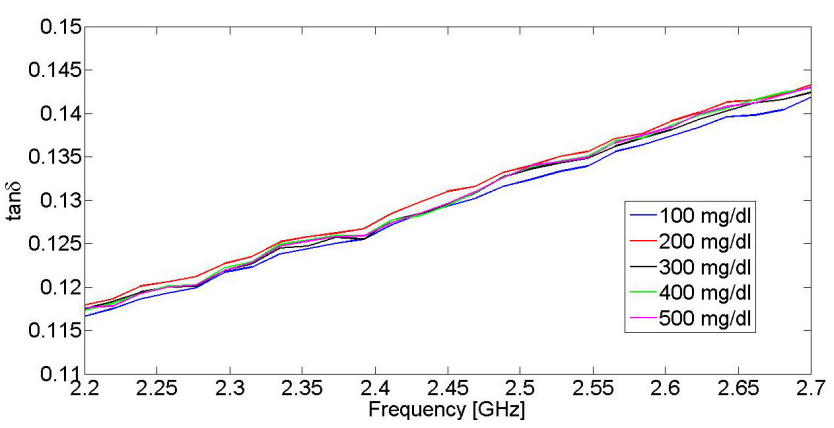

Fig. 4. Measured loss tangent vs. frequency for water solutions with different glucose concentrations.

error less than $1 \%$. A photograph of the adopted test setup is illustrated in Fig. 2, while the measured frequency data of both dielectric constant as well as loss tangent are reported in Figs. 3 and 4, respectively.

From data represented in Figs. 3 and 4, it is straightforward to deduce that both real and imaginary parts of permittivity for water-glucose solutions are influenced by the glucose concentration, thus both information should be considered as input data in the synthesis process of the microwave resonant sensor. Furthermore, the dependency of both dielectric constant and loss tangent on the glucose level can be fruitfully considered to elaborate reliable correlations from which the glucose level can be accurately retrieved. On the basis of the above considerations, measured complex permittivity of water-glucose solutions, retrieved from the preliminary dielectric characterization 
stage, is used as input data in the synthesis procedure of the microwave sensor, acting to obtain the antenna dimensions which guarantee the optimum matching condition at the design frequency $f_{\mathrm{o}}=2.4 \mathrm{GHz}$.

Reflection measurements are then performed on the water solutions with different GC (the same as those adopted for complex permittivity characterization), and the experimental return loss curves are reported in Fig. 5, where a photograph of the realized microwave sensor is also illustrated. To avoid inaccuracies due to the positioning of the microwave sensor, a specific plexiglass box including a slit is adopted to contain the measured waterglucose solutions, as illustrated in Fig. 6.

Two significant observations can be done with reference to measured data of Fig. 5, namely:

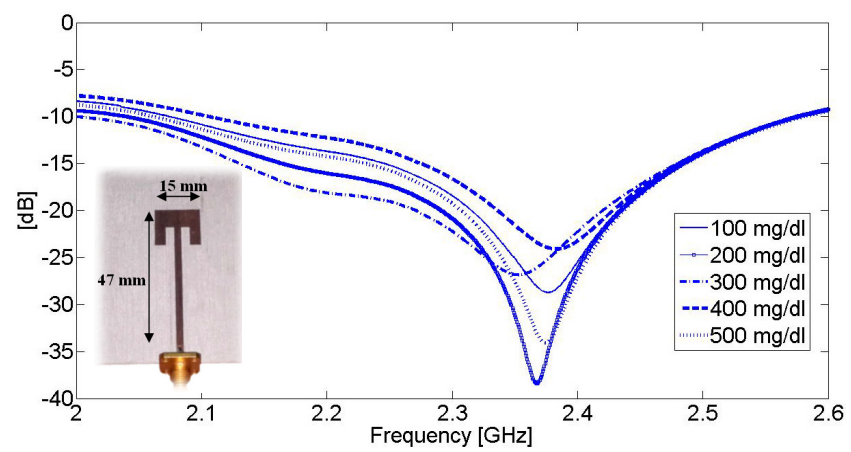

Fig. 5. Measured return loss of microwave sensor for various GC (realized prototype is visible on the lower left corner).

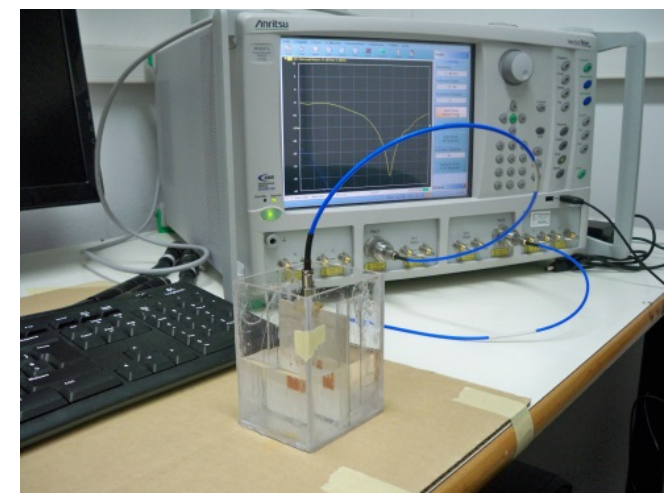

Fig. 6. Test setup for return loss measurements.

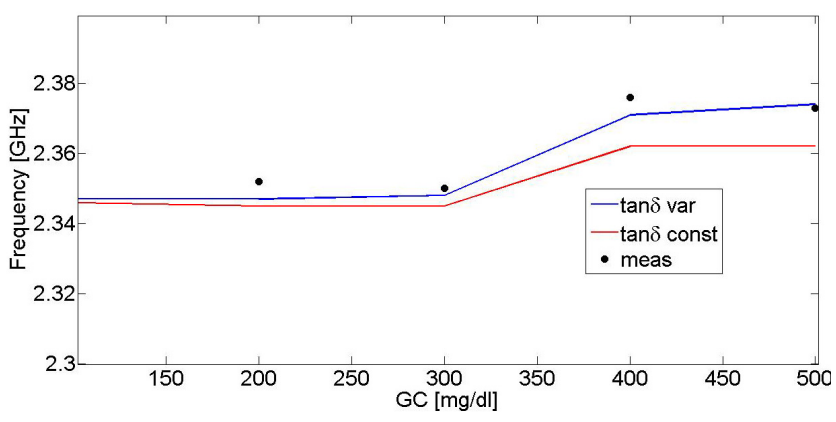

Fig. 7. Frequency variation vs. GC in the reflection response of microwave sensor.
- a frequency shift is clearly visible when varying the $\mathrm{GC}$; in the existing works [5], [10-12], this is the only effect considered till now for the retrieval of glucose level;

- an amplitude variation in the curves is obtained for the different GC; this effect, related to the variation of loss tangent, is not actually taken into account in literature, and it could be strongly useful to elaborate future enhanced correlation models between the reflection response of resonant microwave sensor and the relative GC.

Finally, in Fig. 7, the frequency variation versus GC is reported, by comparing measured data of Fig. 5 with the simulated results (Ansys software) obtained in two distinct cases, namely with and without assuming the loss tangent variation. The comparison clearly demonstrates a more accurate result when the loss tangent variation is considered in the synthesis process of the microwave sensor.

\section{Conclusion}

The importance and physical principles related to the adoption of microwave and millimeter waves for non-invasive monitoring of biological parameters have been discussed in the present work. Existing limitations in the design criteria of microwave biosensors have been clearly outlined, together with new useful perspectives for improving the accuracy of future implementations. The importance of assuming the loss tangent frequency variation of the biological radiation medium in the design of microwave sensor is particularly highlighted, and validation results in the framework of glucose level monitoring have been discussed. The concepts outlined in the present work are intended to provide useful starting guidelines in the implementation of reliable non-invasive monitoring systems based on microwaves or millimeter waves as excitation source for biomedical applications.

\section{References}

[1] NEHRING, J., NASR, I., BORUTTA, K., WEIGEL, R., KISSINGER, D. A silicon integrated microwave vector network analyzer for biomedical sensor read-out applications. In Proceedings of IEEE MTT-S International Microwave Symposium. Tampa (FL), 2014. DOI: 10.1109/MWSYM.2014.6848254

[2] KOELPIN, A., VINCI, G., LAEMMLE, B., KISSINGER, D., WEIGEL, R. The six port in modern society. IEEE Microwave Magazine, 2010, vol. 11 , no. 7 , p. 35-43. DOI: 10.1109/MMM.2010.938584

[3] ROSEN, A., STUCHLY, M. A., VANDER VORST, A. Applications of $\mathrm{RF} /$ microwaves in medicine. IEEE Transactions on $\mathrm{Mi}$ crowave Theory and Techniques, 2002, vol. 50, no. 3, p. 963-974. DOI: $10.1109 / 22.989979$

[4] GRENIER, K., DUBUC, D., POLENI, P.-E., KUMEMURA, M., TOSHIYOSHI, H., FUJII, T., FUJITA, H. Integrated broadband microwave and microfluidic sensor dedicated to bioengineering. 
IEEE Transactions on Microwave Theory and Techniques, 2009, vol. 57, no. 12, p. 3246-3253. DOI: 10.1109/TMTT.2009.2034226

[5] JEAN, B. R., GREEN, E. C., MCCLUNG, M. J. A microwave frequency sensor for non-invasive blood-glucose measurement. In Proceedings of IEEE Sensors Applications Symposium. Atlanta (GA), 2008. DOI: 10.1109/SAS.2008.4472932

[6] AHMADI, M. M., JULLIEN, G. A. A wireless-implantable microsystem for continuous blood glucose monitoring. IEEE Transactions on Biomedical Circuits and Systems, 2009, vol. 3, no. 3, p. 169-180. DOI: 10.1109/TBCAS.2009.2016844

[7] COLE, K. S., COLE, R. H. Dispersion and absorption in dielectrics I. Alternating current characteristics. Journal of Chemical Physics, 1941, vol. 9, p. 341-351. DOI: 10.1063/1.1750906

[8] GABRIEL, S., LAU, R., W., GABRIEL, C. The dielectric properties of biological tissues: III. Parametric models for the dielectric spectrum of tissues. Physics in Medicine and Biology, 1996, vol. 41, no. 11, p. 2271-2293.

[9] KARACOLAK, T., HOOD, A., TOPSAKAL, E. Design of a dualband implantable antenna and development of skin mimicking gels for continuous glucose monitoring. IEEE Transactions on Microwave Theory and Techniques, 2008, vol. 56, no. 4, p. 1001-1008. DOI: 10.1109/TMTT.2008.919373

[10] VENKATARAMAN, J., FREER, B. Feasibility of non-invasive blood glucose monitoring. In Proceedings of IEEE International Symposium on Antennas and Propagation AP-S/URSI. Spokane (WA), 2011, p. 603-606. DOI: 10.1109/APS.2011.5996782

[11] BAGHBANI, R., RAD, M. A., POURZIAD, A. Microwave sensor for non-invasive glucose measurements design and implementation of a novel linear. IET Wireless Sensor Systems, 2015, vol. 5, no. 2, p. 51-57. DOI: 10.1049 /iet-wss.2013.0099

[12] CHOI, H., NAYLON, J., LUZIO, S., BEUTLER, J., BIRCHALL, J., MARTIN, C., PORCH, A. Design and in vitro interference test of microwave noninvasive blood glucose monitoring sensor. IEEE Transactions on Microwave Theory and Techniques, 2015, vol. 63, no. 10, p. 3016-3025. DOI: 10.1109/TMTT.2015.2472019

[13] COSTANZO, S. Loss tangent effect on the accurate design of microwave sensors for blood glucose monitoring. In Proceedings of the $11^{\text {th }}$ European Conference on Antennas and Propagation EuCAP. Paris (France), March 2017.

\section{About the Author ...}

Sandra COSTANZO received the Laurea degree (summa cum laude) in Computer Engineering from the University of Calabria in 1996, and the Ph.D. degree in Electronic Engineering from the University of Reggio Calabria in 2000. Currently, she is an Associate Professor at the University of Calabria, Italy, where she teaches the courses of electromagnetic waves propagation, remote sensing and radar systems and electromagnetic diagnostics. At the same University, she is the Coordinator of Master Degree Course in Telecommunication Engineering.

Since 1996, she has been involved in many research projects funded by ESA (European Space Agency), ASI (Agenzia Spaziale Italiana), MIUR (Ministero dell'Istruzione, dell'Università e della Ricerca) and private companies. She is a Senior Member of IEEE, member of IEEE South Italy Geoscience and Remote Sensing Chapter, CNIT (Consorzio Nazionale Interuniversitario per le Telecomunicazioni) and SIEm (Società Italiana di Elettromagnetismo), and a Board Member of IEEEAP/ED/MTT North Italy Chapter, and of IEEE Information Theory Italy Chapter. She is an Associate Editor of IEEE Antennas and Wireless Propagation Letters, IEEE Access and Radioengineering journals.

Her research interests are focused on near-field far-field techniques, antenna measurement techniques, antenna analysis and synthesis, numerical methods in electromagnetics, millimeter-wave antennas, reflectarrays, synthesis methods for microwave structures, electromagnetic characterization of materials, innovative antennas and technologies for radar applications.

She has been Editor of two books and Lead Editor of three special issues on international journals. She has (co) authored more than 160 contributions in international journals, books and conferences. 